\title{
Modelling and Control of Multi-type Grid-scale Energy Storage for Power System Frequency Response
}

\author{
Saif Sabah Sami, Student member, IEEE, Meng Cheng and Jianzhong Wu, Member, IEEE \\ Cardiff School of Engineering, Cardiff University \\ Cardiff, UK \\ Samiss@cardiff.ac.uk, Chengm2@cardiff.ac.uk,Wuj5@cardiff.ac.uk
}

\begin{abstract}
As a result of the increasing integration of renewable energy sources, power system is changing to a low inertia system with intermittent power supply. Frequency stability is therefore difficult to be maintained. Rather than increasing the spinning reserve capacity from conventional fossilfuel generators, the use of Energy Storage System (ESS) for frequency response is considered as a technically viable lowcarbon solution. To facilitate the grid-level study which aggregates a number of wide-spread small-size ESS, simplified models of multi-type ESSs including batteries and flywheels were developed. A generalized frequency controller was developed and applied to the aforementioned types of ESS. The controller coordinates the response amongst a population of ESS based on the units' State of Charge indicator. An adaptive droop control is combined with the coordinated control to guarantee a linear frequency response provided by a smaller number of ESS units. The number of charging and discharging of each unit is therefore reduced which prolongs the lifetime of the ESS units. Case studies were carried out by connecting a number of multi-type ESSs to a simplified GB power system model. Results show that the grid-scale ESSs are able to provide frequency response similar to but faster than frequency-sensitive generators. Implementation of ESS is therefore technically feasible to support the grid frequency stability with the reduction in the spinning reserve capacity.
\end{abstract}

Keywords- Flywheel energy storage system, Battery energy storage system, Adaptive droop control, Decentralised control, Frequency Response

\section{INTRODUCTION}

The power system security encounters greater challenges as a result of the increasing integration of Renewable Energy Sources (RES). The intermittency of RES causes difficulties to control and dispatch the power generated by RES. Consequently, maintaining the power system frequency stability is more challenging. However, frequency is mandatory to be maintained within certain boundaries. In case of a sudden generation trip, a severe drop in frequency may occur. The power generated from RES is non-despatchable and hence has limited capability to provide response or reserve in case of system disturbances. In addition, RES is connected to the power system through power electronics which reduces the system inertia. Therefore, fast committing units are necessary to restore the frequency and hence to avoid the occurrence of

The work was supported in part by Higher Committee for Education Development in Iraq (HCED), by RESTORES project under the grant of EPSRC and by P2P-SmarTest project under the grant of EU commission the blackout. Rather than frequency-sensitive generators, faster committing units, such as the Energy Storage System (ESS) and demand response, are anticipated [1].

ESS emerged rapidly as a solution for the aforementioned challenges in the power system. ESS converts surplus electricity to a different energy form to be stored, and the stored energy reverts back to electricity according to system needs. The conversion and response are fast compared with conventional generators and therefore mitigate the impact of the reduced system inertia. A study in [2] expected that the deployment of ESS in the Great Britain (GB) power system by 2050 will be 25 GW based on the 'Gone Green Scenario'.

ESS can be classified into chemical, electrical, mechanical and thermal ESS based on the form of energy it converts from/to. Likewise, ESS can also be classified into high power density and high energy density [3]. High power density ESS (e.g. flywheels, super-capacitors, batteries) is able to provide high power output immediately (e.g. in milliseconds) and is suitable to improve the power quality such as the provision of frequency response and the mitigation of the RES power output fluctuations. Contrarily, high energy density ESS (e.g. hydro pumped storage, compressed air) requires longer time to commit once instructed and therefore is mainly used for energy management including the provision of load leveling and the relief of the transmission congestions.

In this paper, two types of high power density ESS were modelled and controlled for the provision of continuous frequency response in the GB power system. Simplified models of both the Battery Energy Storage Systems (BESS) and the Flywheel Energy Storage System (FESS) were developed. A generalised decentralised frequency control was developed for the multi-type grid-scale ESSs and was applied to the ESS models for validation. The performance of the proposed generalised control scheme was compared with the traditional droop control by connecting the ESS models to a simplified GB power system model. The main advantage of the proposed control is then summarised.

\section{MODELLING OF DIFFERENT TYPES OF ESS}

In this research, two types of ESS (i.e. FESS and BESS), which are commercially feasible for grid frequency response, was chosen to be modelled and controlled. 


\section{A. Battery Energy Storage System Model}

Battery Energy Storage System (BESS) stores energy in multiple cells. These cells can be connected in series, in parallel or in both in order to obtain the desired capacity and voltage. Currently, five types of batteries (Lithium-Ion, LeadAcid, Sodium-Sulphur, Nickel-Cadmium and Flow-type) have usually been utilized for power system applications [3].

In this paper, a simplified BESS model is developed. The model consists of the power electronic converters model and the generic battery model (Fig. 1). The model of the power electronic converters is simplified as a first order lag (converters in Fig. 1) which represents the delays in the control of converters on a given reference power $P_{\text {ref }}$ (Watts). The analytical equation is given by (1). $T_{\text {delay }}(\mathrm{s})$ is the time constant of the power converter control loop and $P_{\text {Batteries_in }}(\mathrm{W})$ is the input power to the generic battery model. $P_{\text {Batteries_in }}=P_{\text {ref }}\left(\frac{1}{1+s T_{\text {delay }}}\right)$

The generic battery model ('Module (cells)' in Fig. 1) is composed of a controllable voltage source, controllable current source and a resistance connected in series [4]. The charging and discharging characteristics were assumed similar. The battery no load voltage $\left(E_{r e f}\right)$ is obtained based on the State of Charge (SoC) using (2) [4], in which SoC is calculated using a function of the battery current $(i)$ in (3) [5]:

$$
E_{r e f}=E_{0}-K \frac{Q}{Q-\int i . d t}+A \exp \left(-B \int i d t\right)
$$

$\operatorname{SoC}=1-\frac{\int i d t}{Q}$

where $E_{o}(\mathrm{~V})$ is the battery constant voltage, $K(\mathrm{~V})$ is the polarization voltage, $Q(\mathrm{Ah})$ is the battery capacity, $A(\mathrm{~V})$ is the exponential zone amplitude of the battery and $B\left(\mathrm{Ah}^{-1}\right)$ is the inverse of the exponential zone time constant.

The generic battery model has been validated by comparing the discharging curves with the manufacturer discharging chart [6]. The model performance matches closely to the manufacturers' charts (Fig. 2).

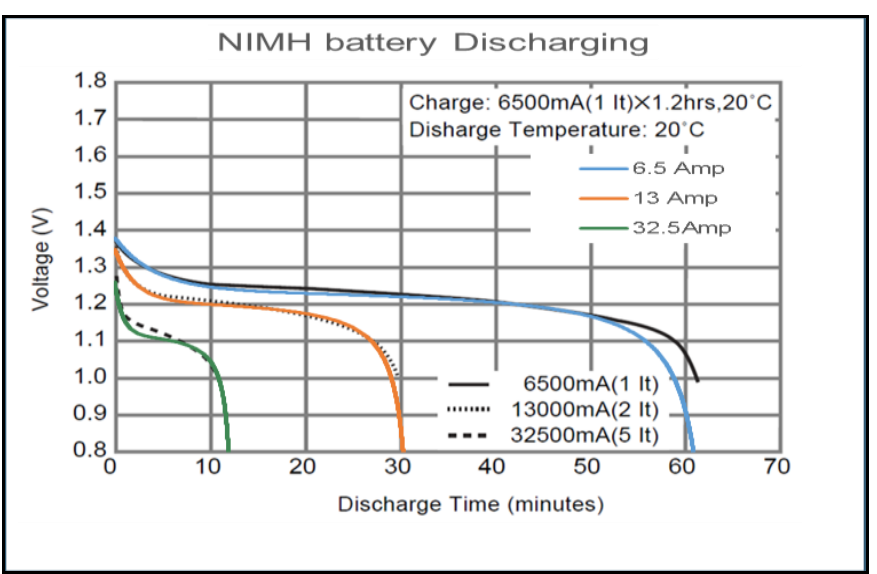

Fig. 2 Battery model validation

\section{B. Flywheel Energy Storage System Model}

Flywheel Energy Storage System (FESS) stores mechanical energy in a rotating flywheel that is coupled with an electrical machine. When the flywheel is increasing the rotating speed, the electrical energy is converted to mechanical energy. Alternatively, the electrical energy is restored by reducing the rotating speed.

A simplified model of FESS was developed. FESS is composed of the model of power electronic converters and the model of the electromechanical parts. Similar to the BESS model, a first order lag was used to model the converters (Fig. 3 ). The mechanical part of a FESS is modelled using the following electromechanical equation:

$J \frac{d \omega}{d t}=-T_{\text {in }}=-\frac{P_{\text {elec_in }}}{\omega}$

where $J\left(\mathrm{kgm}^{2}\right)$ is the flywheel inertia, $\omega(\mathrm{rad} / \mathrm{s})$ is the rotating speed, $T_{\text {in }}(\mathrm{Nm})$ is the input torque of the flywheel, $P_{\text {elec } \_ \text {in }}(\mathrm{W})$ is the output power controlled by converters in Fig. 3. The simplified model of FESS has been validated with a detailed FESS model which includes the flywheel, the electrical machine and the back-to-back converters [7][8].

The simplified model provided accurate results with significant reduction in computational time and hence is used for grid-level studies.

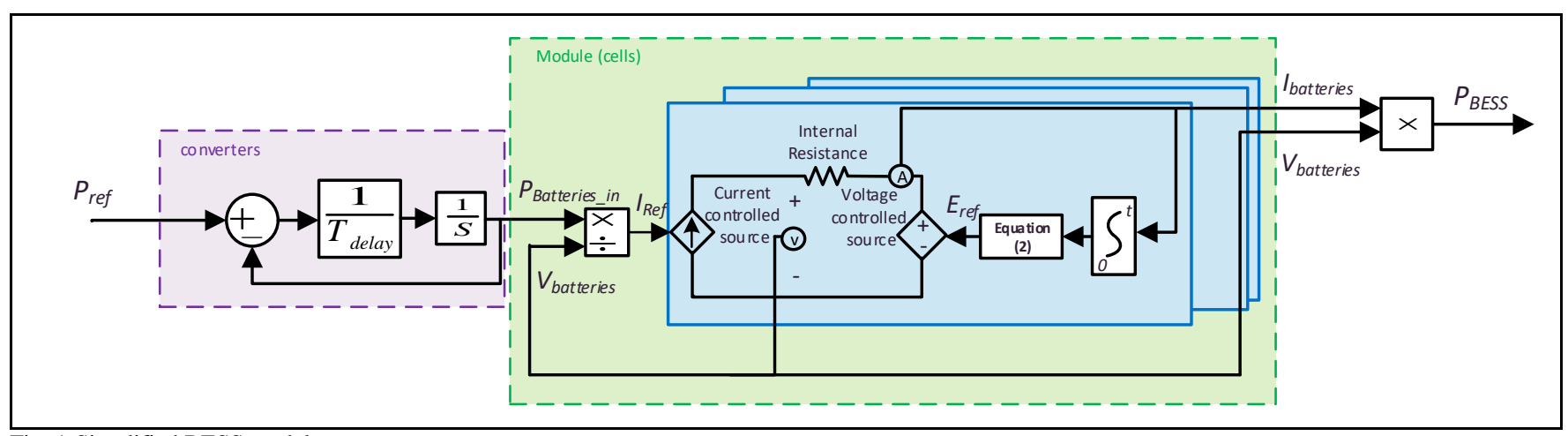

Fig. 1 Simplified BESS model 


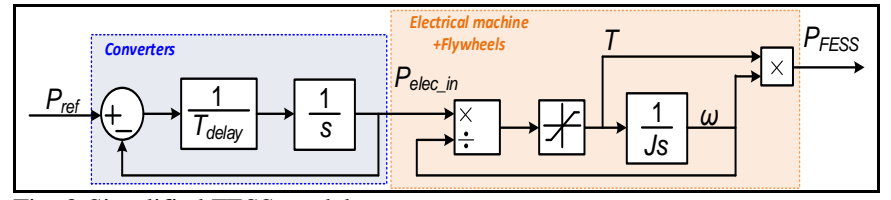

Fig. 3 Simplified FESS model

\section{GENERALISED FREQUENCY CONTROL}

\section{A. Conventional droop control (CDC)}

The Conventional Droop Control (CDC) is widely used by ESS to provide frequency response. Following the changes in frequency $(d f)$, all ESS units change their power output $(d P)$ according to (5):

$$
d P=\frac{d f}{R}
$$

For BESS and FESS, $\mathrm{R}$ is set to $1 \%$ which provides $100 \%$ power output change when frequency variations are equal to or higher than $1 \%$ (i.e. $0.5 \mathrm{~Hz}$ ).

\section{B. Coordinated Constant Droop Control (CCDC)}

A method of coordinating ESS units' response based on the SoC of each individual unit, namely, a Coordinated Constant Droop Control (CCDC) was developed. Rather than all units keep responding to frequency changes, CCDC allows that the more the frequency deviates, the greater number of ESS units will respond.

The generalized frequency control was developed as shown in Fig. 4. The control constantly measures the grid frequency $f$. The control output is the final required power of BESS or FESS units. Each storage unit is assigned a pair of trigger frequency, namely $F_{O N}$ and $F_{O F F}$. Consistently with the steadystate limits of grid frequency in the GB power system, the range of $F_{O N}$ is $50-50.5 \mathrm{~Hz}$ and the range of $F_{\text {OFF }}$ is $49.5-50$ $\mathrm{Hz}$.

If $f$ is higher than $F_{O N}$ of a unit, the unit will be committed and charge as a result of the frequency rise. If $f$ is higher than $50 \mathrm{~Hz}$ but lower than $F_{O N}$, the unit will standby. If $f$ is lower than $F_{O F F}$, the unit will be committed and discharge as a result of the frequency drop. If $f$ is lower than $50 \mathrm{~Hz}$ but higher than $F_{O F F}$, the unit will standby. $F_{O N}$ and $F_{O F F}$ vary linearly with SoC of each unit as shown in Fig. 4. Specifically for FESS, $\omega$ indicates SoC and for BESS the current indicates SoC. When $f$ rises, the units will start charging from the one with the lowest SoC. When $f$ drops, the units will start discharging from the one with the highest SoC. This is achieved by the linear relationship between the trigger frequencies and SoC. Hence, the more $f$ drops, the more FESS and BESS units will be committed. Vice versa for $f$ rises. The inherent control in Fig. 4 ensures that $\mathrm{SoC}$ stays within its maximum and minimum limits for each unit. The final state of a unit is therefore determined by a set of logic gates. The output of the logic gates decides to charge/discharge the unit or to maintain the unit at the standby mode. Once an ESS unit starts to charge/discharge, the change of the power output is determined by the droop control according to (5) as illustrated in Section III.A and shown in Fig. 4.

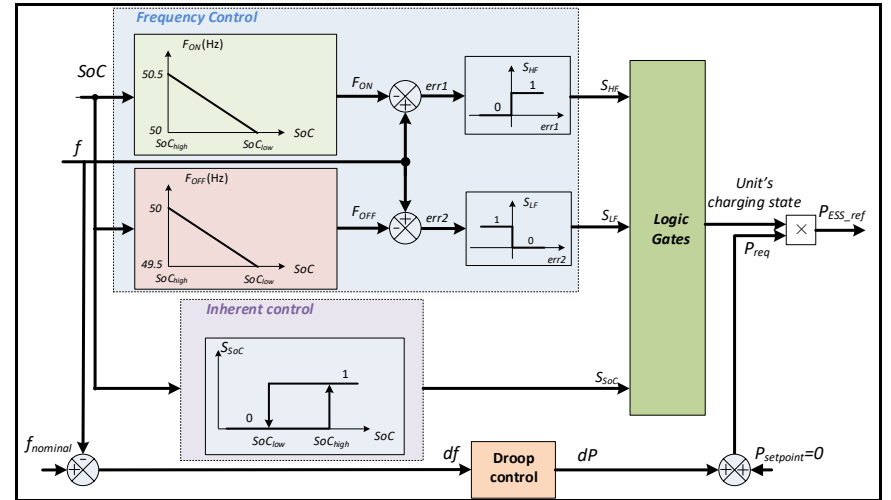

Fig. 4 Coordinated frequency control

The control in Fig. 4 is a local control on each unit, but is coordinated by assigning the trigger frequencies based on SoC. This reduces the number of charging and discharging cycles and each unit has equal opportunity to charge/discharge. The lifetime of units is hence prolonged.

However, when the frequency deviation is small, few number of ESS units are committed. The aggregated change of power output of ESS units is less than that of using CDC where all units are committed. Only when a large frequency deviation occurs, the frequency response level from the CCDC matches the frequency response level from the CDC.

A test frequency varying between below $49.5 \mathrm{~Hz}$ and $50 \mathrm{~Hz}$ was used to examine the number of committed units in response to frequency variations in the CCDC as shown in Fig. 5 . The test validates that the number of committed units varies in linear with the frequency changes.

\section{Coordinated Adaptive Droop Control (CADC)}

In order to provide a similar level of frequency response with CDC while reducing the number of committed units, a Coordinated Adaptive Droop Control (CADC) was developed. The number of committed units changes linearly with frequency deviations, therefore the droop control in Fig. 4 is replaced by an adaptive droop control. Small frequency deviations prompt few committed units. A high droop value $\left(R_{a}\right)$ is then applied to provide more power change from these committed units. When the frequency deviation is large, the droop equals to the conventional droop value $R$ in (5). Therefore, the adaptive droop constantly changes with frequency deviations as shown in (6):

$$
R_{a}=\frac{d f_{\max }}{d f} \times R
$$

where $d f$ is the frequency deviation from the nominal value (i.e. $50 \mathrm{~Hz})$ and $d f_{\max }$ is the frequency deviation limit $(0.5 \mathrm{~Hz}$ in the GB power system).

\section{RESULTS}

A simplified GB power system model [9] was used to assess the proposed control of BESS and FESS. A low frequency incident caused by a consecutive sudden loss of two generators of $345 \mathrm{MW}$ and $1237 \mathrm{MW}$ [9] was undertaken 
consolidated with a part of the GB power system frequency profile with small frequency variations around $50 \mathrm{~Hz}$ [10].

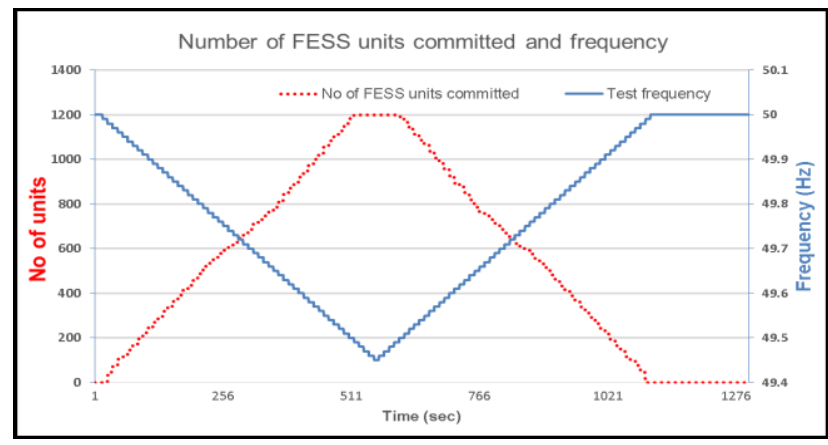

Fig. 5 No. of units committed and frequency correlation

Based on a total demand of $40 \mathrm{GW}$, the system inertia was estimated to be $4.5 \mathrm{~s}$. $10 \mathrm{MW}$ of FESS (400 units each with power rating of $50 \mathrm{~kW}$ ) and $10 \mathrm{MW}$ of NIMH BESS (410 units with $48 \mathrm{~kW}$ power rating) were used to provide continuous frequency support over 30 minutes. Three case studies were carried out:

Case 1: The BESS and FESS are implemented with the Conventional Droop Control (CDC)

Case 2: The BESS and FESS are implemented with the Coordinated Constant Droop Control (CCDC)
Case 3: The BESS and FESS are implemented with the Coordinated Adaptive Droop Control (CADC)

Fig. 6 and Fig. 7 show the frequency and the power output of the BESS and FESS. When frequency is close to $50 \mathrm{~Hz}$, ESSs with the CDC or the CADC show a power output higher than with the CCDC. The power output with either CDC or CADC was similar while only less than one third of units respond in the CADC strategy compared with the $\mathrm{CDC}$ strategy (Fig. 8). This is preferable to extend the lifetime of the costly ESS. In case of the severe frequency drop, ESS with all control strategies has the same level of power output and similar number of unit respond.

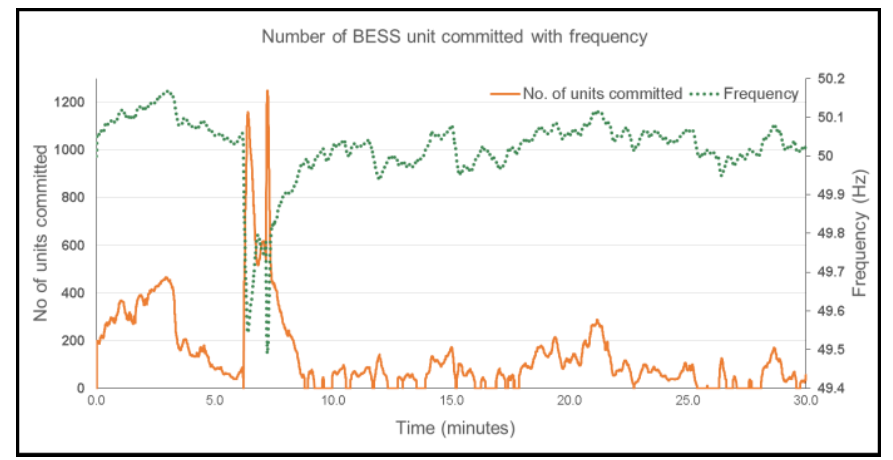

Fig. 8 Number of BESS units committed with frequency

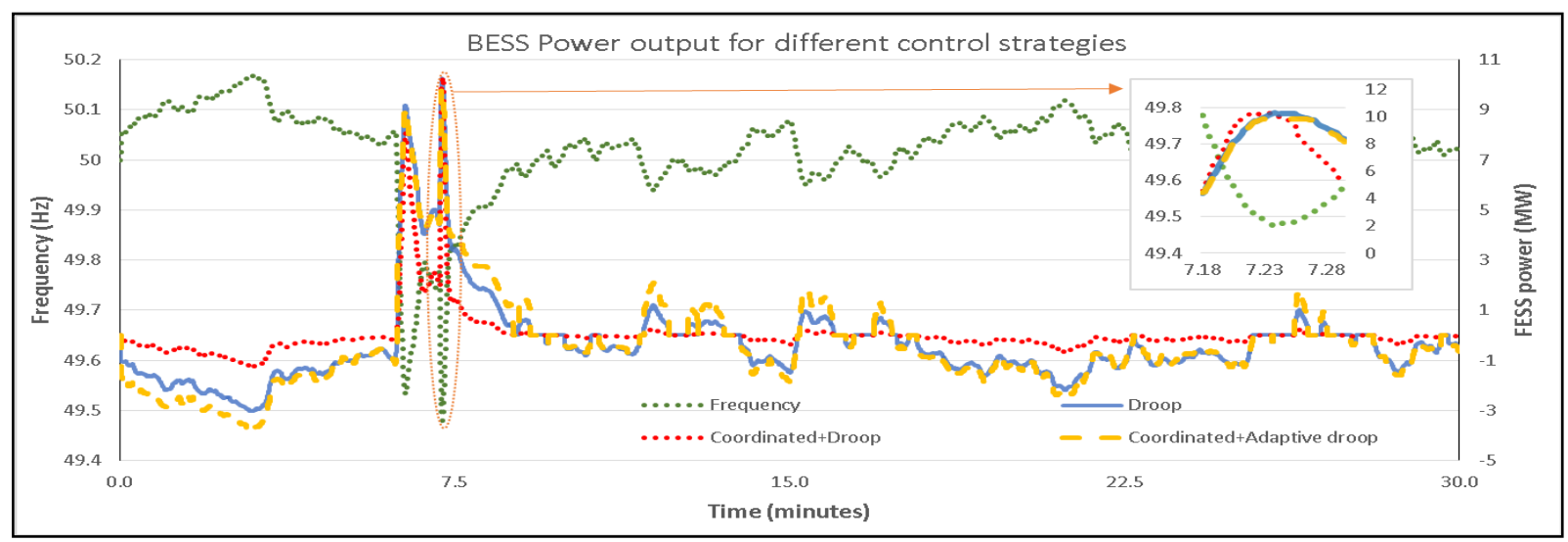

Fig. 6 BESS power output for different control strategies

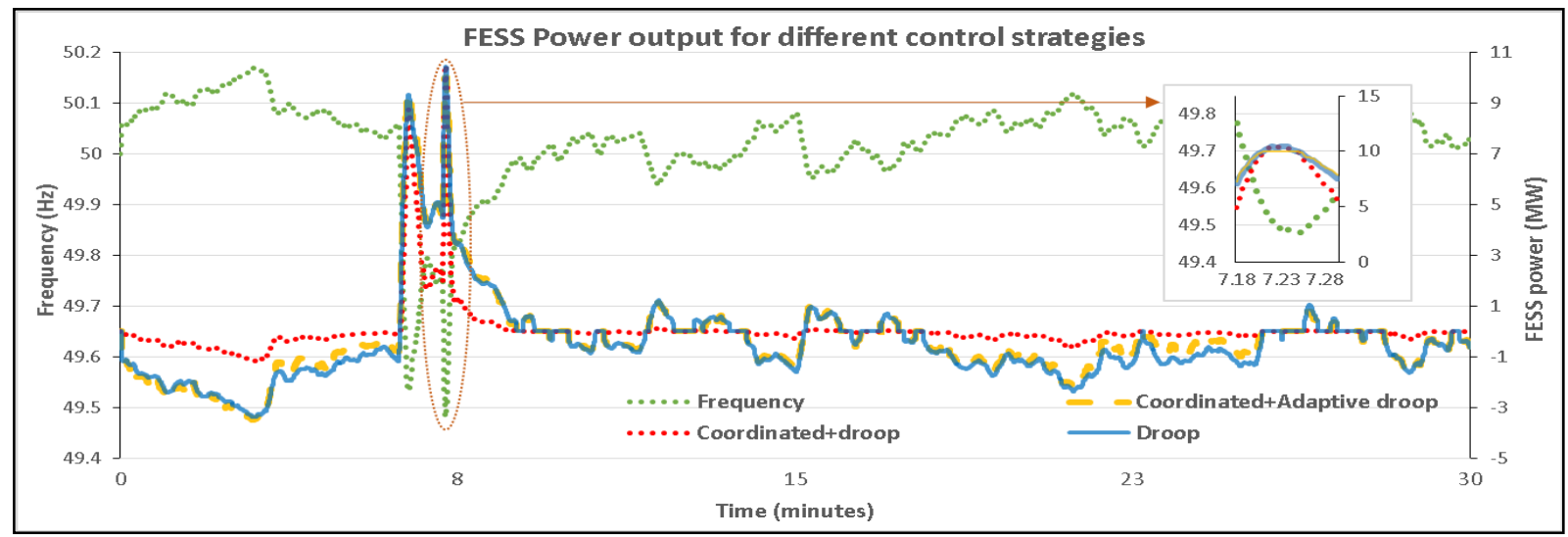

Fig. 7 FESS power output for different control strategies 
In Fig. 9, the number of charging and discharging times that ESSs respond to frequency variations for different control strategies was compared. CADC has an outstanding performance in reducing the number of charging and discharging times compared with $\mathrm{CDC}$ while retaining more power output than CCDC.

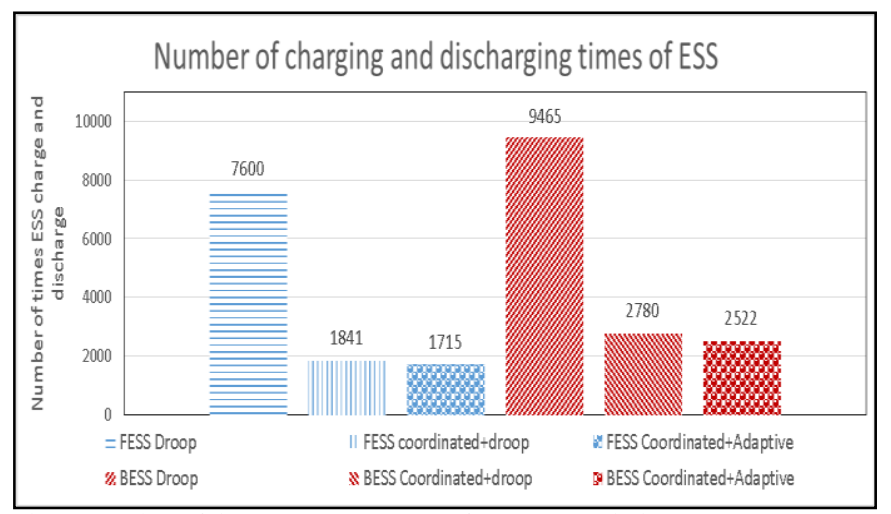

Fig. 9 Number of times ESS response to frequency variation

\section{CONCLUSIONS}

Simplified models of BESS and FESS were developed to facilitate grid scale studies. Two types of control of ESSs, namely, the Coordinated Constant Droop Control (CCDC) and Coordinated Adaptive Droop Control (CADC) were developed. These coordinated control strategies reduce the number of committed units compared to the Conventional Droop Control (CDC) where all units have to be committed. Therefore, the lifetime of each ESS unit is prolonged.

Case studies were carried out by connecting the ESSs to a simplified GB power system model to evaluate the performance of the three control strategies. CADC shows the best performance which provides a similar level of frequency response to $\mathrm{CDC}$ with a much smaller number of committed units.

Therefore, high power density ESSs, such as FESS and BESS, is a technically feasible means to support the grid frequency stability and provide primary, secondary and enhance frequency response services with the reduction in the spinning reserve capacity in the future power system. The CADC shows a potential of increasing the lifetime of the frequent charging and discharging ESS units and hence reduces the long term investment costs.

\section{REFERENCES}

[1] "Firm Frequency Response | National Grid." [Online]. Available:

http://www2.nationalgrid.com/UK/Services/Balancing -services/Frequency-response/Firm-Frequency-

Response/. [Accessed: 28-Jan-2016].

[2] G. Strbac, M. Aunedi, D. Pudjianto, P. Djapic, F. Teng, A. Sturt, D. Jackravut, R. Sansom, V. Yufit, and N. Brandon, "Strategic assessment of the role and value of energy storage systems in the UK low carbon energy future," Rep. Carbon ..., p. 9, 2012.
[3] X. Luo, J. Wang, M. Dooner, and J. Clarke, "Overview of current development in electrical energy storage technologies and the application potential in power system operation," Appl. Energy, vol. 137, pp. 511-536, Oct. 2014.

[4] O. Tremblay, "A generic battery model for the dynamic simulation of hybrid electric vehicles," Veh. power ..., 2007.

[5] S. Teleke and M. Baran, "Optimal control of battery energy storage for wind farm dispatching," Energy Conversion, ..., 2010.

[6] Panasonic, "nickel metal hydride handbook," 2003. [Online]. Available: http://www.rosebatteries.com/pdfs/Panasonic_NiMH_ Hdbk_02-03_v1.pdf. [Accessed: 03-Feb-2016].

[7] C. Chapelsky, J. Salmon, and A. Knight, "Control of a High-Inertia Flywheel As Part of a High Capacity Energy Storage System," in 2007 Canadian Conference on Electrical and Computer Engineering, 2007, pp. 1437-1440.

[8] F. Diaz-Gonzalez, A. Sumper, O. Gomis-Bellmunt, and R. Villafafila-Robles, "Modeling and validation of a flywheel energy storage lab-setup," in Innovative Smart Grid Technologies (ISGT Europe), 2012 3rd IEEE PES International Conference and Exhibition on, 2012, pp. 1-6.

[9] K. Samarakoon, J. Ekanayake, and N. Jenkins, "Investigation of domestic load control to provide primary frequency response using smart meters," Smart Grid, IEEE Trans., vol. 3, no. 1, pp. 282-292, 2012.

[10] "Enhanced Frequency Response | National Grid." [Online]. Available: http://www2.nationalgrid.com/Enhanced-FrequencyResponse.aspx. [Accessed: 28-Jan-2016]. 